\title{
$\underline{065}$
}

\section{Media properties of different vermicompost and coir dust mixtures}

\author{
B W N Priyadarshani', S Senarathne ${ }^{2}$, N A K de Silva ${ }^{2}$ and W G D Lakmini ${ }^{1}$ \\ ${ }^{1}$ Department Crop Science, University of Ruhuna, \\ ${ }^{2}$ Agronomy Division, Coconut Research Institute, Lunuwilla, Sri Lanaka
}

\begin{abstract}
In recent years, the demand for coir dust has increased as it is widely used as a growth medium in soil less culture. Since it is low in nutrients, when mixed with vermicompost, will provide a better growth medium for plant establishment. Therefore, this study was carried out to evaluate the properties of different vermicompost and coir dust mixtures. Tomato was used as the test plant and four different media compositions were tested with and without nutrient supplement. Media properties including chemical (N, P, K, Ca, and $\mathrm{Mg}$ levels, $\mathrm{pH}$ and Electrical conductivity [EC]) and physical properties (Bulk density, Field capacity and drying pattern) were measured in the time period.
\end{abstract}

Nutrient levels, pH, EC, bulk density, and drying rates were found to be decreased and only field capacity increased with increasing the proportion of coir dust. According to the result obtained, vermicompost alone can be used as a plant growth medium. But poor porosity and aeration of vermicompost limit the root growth and lowered the water holding capacity. Therefore, medium with $75 \%$ vermicompost and $25 \%$ coir dust is more suitable than vermicompost alone. Medium with $50 \%$ vermicompost and 50\% coir dust has better physical properties, but nutrient level is not sufficient to support plant growth. Therefore nutrient supplement is needed to use it as growing medium.

\section{$\underline{066}$ \\ Suitability of some agro-industrial by-products as litter material for poultry: Physio-chemical properties}

\author{
N S B M Atapattu, D Senaratne and N D C Sirisena \\ Departement of Animal Science, University of Ruhuna, Sri Lanka.
}

Paddy-husk (PH) based deep litter system is the most popular poultry management system in Sri Lanka. Physio-chemical properties of PH make it an ideal litter material for poultry keeping. Since $\mathrm{PH}$ is increasingly been demanded by other industries, availability of PH for poultry industry has been restricted from recent years. Objective of the present study was to screen the physio-chemical properties of some commonly available agro-industrial by-products, to be used as alternative litter materials for poultry. Physio-chemical properties such as dry matter (DM), bulk density (BD), pH, water absorbability (WA) and water releasing capacity (WRC) of seven alternatives such as rice straw (RS), coir dust (CD), wood shawing;WS, saw dust;SD, tree litter;TL, shredded paper;SP and refused tea;RT)) were determined and, compared with the respective values of $\mathrm{PH}$. The experiment followed a completely randomized design. The DM \% of the alternatives tested ranged from $91 \%$ (SD) to $92 \%$ (SP). DM contents of WS and RT were statistically similar to that of PH. However, none of the material had either too low or too high DM levels to be disqualified as a litter material. $\mathrm{pH}$ values of the tested materials were statistically similar and were slightly acidic (mean 6.27; range 6.1-6.5). PH had the highest BD $\left(0.09 \mathrm{~g} / \mathrm{cm}^{3}\right)$. The BDs of CD, SD and RT, though were lower than $\mathrm{PH}$, the values were not significantly different from that of $\mathrm{PH}$. WA was also statistically different between the materials. CD had the highest $(1101 \%)$ WA while RT showed lowest $(70 \%)$ WA. SD, SP and TL showed statistically similar WA as PH. Materials showed a great variability (from $7.7 \%$ for $\mathrm{SD}$ to $26.7 \%$ for RS) in relation to WRC. PH had $13.6 \% \mathrm{WRC}$ within $24 \mathrm{hrs}$. RT had the closest WRC capacity $(17.9 \%)$ to PH. Materials such as RS, TS, WS and SP showed as high as $25 \%$ WRC. When materials were compared for the parameters tested against those of PH, RS and WS were similar to $\mathrm{PH}$ only in relation to two parameters. Being comparable with $\mathrm{PH}$ for four parameters, SD was identified as the best alternative for PH. CD, TL, SP and RT were comparable with PH in relation to three parameters. Since CD had excessive WA $(1101 \%)$ and very low WRC it can not be recommended further studies. This study concludes that physio-chemical properties of SD, TL, SP and RT are comparable with PH. Field studies involving live poultry are warranted to determine how birds perform and behave when raised on these litter materials.

Proceedings of the International Forestry and Environment Symposium 2006 of the

Department of Forestry and Environmental Science, University of Sri Jayewardenepura. Sri Lanka 